\title{
ON THE CONVERGENCE OF INFINITE EXPONENTIALS
}

\author{
DONALD L. SHELL 1
}

Given a sequence of complex numbers $\left\{a_{i}\right\}$, we define a sequence of functions:

$$
a_{1}^{z}, \quad a_{1}^{\left(a_{2}^{2}\right)}, \quad a_{1}^{\left(a_{2}^{\left(a_{8}\right)}\right)}, \ldots
$$

This sequence is formally represented by $E\left(a_{1}, a_{2}, a_{3}, \cdots ; z\right)$, and is called an infinite exponential. If all the $a_{i}=a$, as is the case in this paper, the infinite exponential is represented by $E(a ; z)$. This nomenclature follows Barrow [1]. Another symbolism has been used by Thron [9]. It is necessary to specify the values $\log a_{n}$ to make the sequence determinate.

Euler [4] was the first to investigate seriously the convergence of the sequence $E(a ; z)$. He stated and demonstrated the results for the case where $a$ and $z$ are real. However, his demonstration was not a rigorous proof. Later proofs of these results were given independently by Seidel [8], Gravé [5], and Barrow [1]. We quote the result that $E(a ; 1)$ converges when

$$
e^{-e} \leqq a \leqq e^{1 / e}
$$

Convergence for complex $a_{i}$ has been considered by Thron [9]. He showed that $E\left(a_{1}, a_{2}, a_{3}, \cdots ; 1\right)$ will converge if $\left|\log a_{i}\right| \leqq e^{-1}$, $i=1,2,3, \cdots$. The purpose of this paper is to establish the existence of a region of convergence extending outside this one in the special case that $a_{i} \equiv a$. If $E(a ; z)$ converges to the limit $t$, then clearly

$$
a^{t}=t \quad \text { or } \quad a=t^{1 / t} \text {. }
$$

If now one lets $t=e^{\zeta}$ where $\zeta$ is a complex parameter, then [2]

$$
a=e^{5 e^{-\zeta}} \text {. }
$$

In the remainder of this paper, the representation (1) will be employed.

Presented to the Society, April 17, 1959; received by the editors March 4, 1959 and, in revised form, July 14, 1959, September 2, 1960, and October 3, 1961.

1 The work reported here was performed in partial fulfillment of the requirements for the Ph.D. Degree at the University of Cincinnati under the direction of Dr. A. J. Macintyre. Financial support was provided by a Laws Fellowship at the University and a living allowance from the General Electric Company. The author is currently located at GEL, General Electric Company, Schenectady, New York. 
$E(a ; z)$ is the sequence of iterates of the function $\phi(z)$. That is, it is identical with the sequence $\phi_{n}(z)$ defined by

$$
\begin{gathered}
\phi_{1}(z)=\phi(z)=a^{z}, \\
\phi_{2}(z)=\phi\left(\phi_{1}(z)\right)=a^{\phi_{1}(z)}, \\
\phi_{n}(z)=\phi\left(\phi_{n-1}(z)\right)=a^{\phi_{n-1}(z)} .
\end{gathered}
$$

Now $\phi(z)$ is an entire function with the fixed point $z_{0}=e^{\zeta}$ and the theory of iteration $[6 ; 7$, pp. 229-239] tells us that the sequence $\phi_{n}(z)$ will converge for $z$ in some neighborhood of the fixed point provided that $\left|\phi^{\prime}\left(z_{0}\right)\right|<1$.

Now using (1)

$$
\frac{d \phi}{d z}=\frac{d}{d z} e^{\zeta e^{-\zeta z}}=e^{\zeta}-\zeta_{z} \zeta e^{-\zeta}, \quad \frac{d \phi\left(e^{\zeta}\right)}{d z}=\zeta .
$$

Hence we have [2]

THEOREM 1. If $|\zeta|<1$ and $a=e^{\zeta_{e}}-\zeta$, then $E(a ; z)$ converges to $e^{5}$ for $z$ in some neighborhood of $z_{0}=e^{\zeta}$.

The results of this paper are based on the estimate of the convergence neighborhood given in

Theorem 2, If $|\zeta|<1$ and $a=e^{\zeta_{0}}-\zeta$ then $E(a ; z)$ converges to $e^{5}$ provided $\left|z-e^{\zeta}\right|<r_{0}\left|e^{\zeta}\right|$ where $r_{0}$ is the positive root of the equation $r^{-1} \log (1+r)=|\zeta|$.

The convergence is uniform in

$$
\left|z-e^{\xi}\right| \leqq \theta r_{0}\left|e^{\xi}\right| ; \quad 0<\theta<1 .
$$

From the point of view of the theory of iteration of functions $E(a ; 1)$ is merely a special case of $E(a ; z)$. This was recognized by Euler, but as Condorcet [3] originally raised the question of the convergence of $E(a ; 1)$ there is particular interest in determining the values of $a$ for which $E(a ; 1)$ converges. We shall deduce from Theorem 2

Theorem 3. If $a=e^{\zeta_{0}^{-\zeta}}$ and $|\zeta| \leqq \log 2$ then $E(a ; 1)$ converges to $e^{5}$.

A slightly more extensive region than $|\zeta| \leqq \log 2$ will be found as stated below in Theorem 4 .

The regions of convergence of $E(a ; 1)$ in the upper half of the $a$ plane as established by Thron, Theorem 3 and Theorem 4 are illustrated in Figure I which is based on computations performed on an electronic computer. 
Theorem 1 was given by Carlsson [2].

Before proceeding to prove Theorem 2 it is helpful to consider the function

$$
\theta(r)=\frac{e^{r s}-1}{r}=s+\frac{r s^{2}}{2 !}+\frac{r^{2} s^{3}}{3 !}+\cdots
$$

We evidently have the following

LemMa. If $0<s<1$ then $\theta(r)$ is monotone increasing with $r$ for $r>0$. There exists a unique positive solution $r=r_{0}$ of the equation $r^{-1} \log (1+r)$ $=s$ and $\theta\left(r_{0}\right)=1 . s<\theta(r)<1$ for $0<r<r_{0}$.

Now let $z=e^{\zeta}(1+\beta)$. We find that $a^{z}=\exp \left(z \zeta e^{-\zeta}\right)=e^{\zeta} e^{\beta \zeta}$. We propose to establish conditions under which $\left|a^{z}-e^{\xi}\right|<\left|z-e^{\xi}\right|$. Evidently $\left|a^{z}-e^{\zeta}\right|=\left|e^{\xi}\right|\left|e^{\beta \zeta}-1\right| \leqq\left|e^{\zeta}\right|\left(e^{|\beta \zeta|}-1\right)$. Now with $s=|\zeta|$ we find that provided $|\beta| \leqq r$ then

$$
\left|a^{z}-e^{\zeta}\right| \leqq\left|e^{\zeta}\right| \theta(r)|\beta|=\theta(r)\left|z-e^{\zeta}\right| \text {. }
$$

Hence if $|\beta| \leqq r<r_{0}$ we have

$$
\left|\phi_{1}(z)-e^{\zeta}\right|=\left|\beta_{1} e^{\xi}\right| \leqq \theta(r)\left|z-e^{\zeta}\right|=\theta(r)\left|\beta e^{\zeta}\right| .
$$

This implies that $\left|\beta_{1}\right| \leqq \theta(r)|\beta| \leqq \theta(r) r \leqq r$ and hence repeating the argument

$$
\left|\phi_{2}(z)-e^{\xi}\right|=\left|\beta_{2} e^{\xi}\right| \leqq \theta(r)\left|\phi_{1}(z)-e^{\xi}\right| \leqq[\theta(r)]^{2}\left|z-e^{\xi}\right| .
$$

Evidently $\left|\phi_{n}(z)-e^{\zeta}\right| \leqq[\theta(r)]^{n}\left|z-e^{\zeta}\right|$ and $\phi_{n}(z)$ converges uniformly to $e^{s}$ for $\left|z-e^{\xi}\right| \leqq r\left|e^{\xi}\right|$. This proves Theorem 2 .

We prove Theorem 3 by showing that $z=1$ is within the circle of convergence associated with each number $a$ in the relevant region. Substituting $|\zeta|=\log 2$ in equation (2) we find $r_{0}=1$. Hence, we shall have convergence in this case if $|\beta|<1$. The point under consideration is $z=1=e^{\zeta}(1+\beta)$. Here $\beta=e^{-\zeta}-1$ and $|\beta|=\left|e^{-\zeta}-1\right| \leqq e^{|\zeta|}-1$ $\leqq e^{\log 2}-1=1$. Now the equality holds only when $-\zeta=|\zeta|=\log 2$. Otherwise, $|\beta|<1$. But when $\zeta=-\log 2$, we appeal to the results for real $a$, which indicate convergence when $a=1 / 4$ (corresponding to $\zeta=-\log 2$ ). This proves Theorem 3 .

It is clear that equation (2) assures convergence in a somewhat larger region. Thus letting

$$
r=|\beta|=\left|e^{-\zeta}-1\right|
$$

we define a contour in the $\zeta$-plane, namely

$$
|\zeta|=\frac{\log \left(1+\left|e^{-\zeta}-1\right|\right)}{\left|e^{-\zeta}-1\right|}
$$


within which $\left|e^{s}-1\right|<r_{0}\left|e^{s}\right|$. Hence, we have

TheOREM 4. The infinite exponential $E(a ; 1)$ converges to $e^{\zeta}$ when $\zeta$ is within the contour defined by equation (3) and $a=e^{50^{-\zeta}}$.

I wish to thank Dr. A. J. Macintyre for proposing this problem and offering numerous helpful suggestions toward its solution.

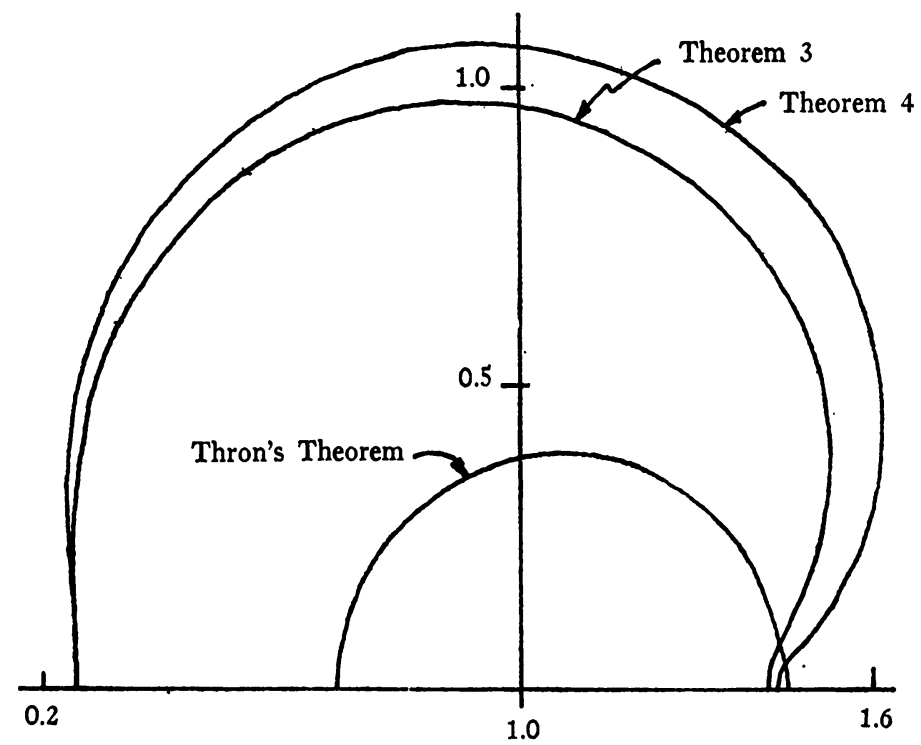

FIGURE 1. Regions of convergence of $E(a ; 1)$.

\section{REFERENCES}

1. D. F. Barrow, Infinite exponentials, Amer. Math. Monthly 43 (1936), 150-160.

2. A. Carlsson, On Itereade Funktioner, Uppsala (1907).

3. Marquis de Condorcet, Sur quelques series infinies dont la somme punt etre exprimee par des fontions analytiques d'une forme particuliere, Acta Academiae Scientiarum Petropolitanae 1 (1777), 34-37.

4. L. Euler, De formulis exponentialibus replicatus, Opera Omnia, Series Prima XV, 268-297; Acta Academiae Petropolitanae 1 (1777), 38-60.

5. M. D. Gravé, Sur les expressions dites surpuissances, Nouvelles Annales de Mathematiques (3) 17 (1898), 80-91.

6. G. Koenigs, Recherches sur les intégrales de certaines equations fonctionnelles, Ann. Sci. Ecole Norm. Sup. (3) 1 (1884), S3-S41.

7. P. Montel, Leçons sur les recurrences et leurs applications, Paris, 1957.

8. L. Seidel, Ueber die Grenzwerthe eines unendlichen Potenzausdruckes, Abhandlungen der Mathematisch physikalischen Classe der Königlich Bayerischen Akademie der Wissenschaften 11 (1874), 1-10.

9. W. J. Thron, Convergence of infinite exponentials with complex elements, Proc. Amer. Math. Soc. 8 (1957), 1040-1043.

General Electric Company, Schenectady, New York 